INPLASY

PROTOCOL

To cite: Almulihi et al. Does Departmental Simulation and Team Training Program Reduce Medical Error and Improve Quality of Patient Care? A Systemic Review. Inplasy protocol 202230006. doi:

10.37766/inplasy2022.3.0006

Received: 02 March 2022

Published: 02 March 2022

Corresponding author:

Qasem Almulihi

qasem.almulihi@hotmail.com

Author Affiliation:

King Fahad University Hospital

- Saudi Arabia.

Support: No.

Review Stage at time of this submission: Completed but not published.

Conflicts of interest:

None declared.

\section{Does Departmental Simulation and Team Training Program Reduce Medical Error and Improve Quality of Patient Care? A Systemic Review}

Review question / Objective: This systematic review aimed to assess whether human simulations or machine stimulations programs would help to prevent medical errors and improve patient safety.

Information sources: The search terms "Medical Simulation" [Mesh], "Medication Errors" [Mesh], "Patient safety" [Mesh] were implemented, to be as specific and selective as possible. We searched for all the publications in the Medline database, Web of Science, and Google Scholar from 2000 (when the idea of simulation in healthcare to prevent ME was employed for the first time by the Institute of Medicine (IOM)) to Feb 2022 with only English language-based literature Electronic databases.

INPLASY registration number: This protocol was registered with the International Platform of Registered Systematic Review and Meta-Analysis Protocols (INPLASY) on 02 March 2022 and was last updated on 02 March 2022 (registration number INPLASY202230006).

\section{INTRODUCTION}

Review question / Objective: This systematic review aimed to assess whether human simulations or machine stimulations programs would help to prevent medical errors and improve patient safety.

Condition being studied: Prevent medical errors and improve patient safety by
Medical training and stimulations programs.

\section{METHODS}

Search strategy: We searched for all the publications in the Medline database, Web of Science, and Google Scholar from 2000 (when the idea of simulation in healthcare to prevent ME was employed for the first time by the Institute of Medicine (IOM)) to 
Feb 2022 with only English language-based literature.

Participant or population: Health professionals, Nurses, Medical Interns, Students.

Intervention: Simulations.

Comparator: Traditional teaching or other stimulations scenario.

Study designs to be included: Clinical Trials and Cohort studies published in English.

Eligibility criteria: We included only those studies conducted in hospitals or universities which met our PICO criteria and focused on human simulation such as prescribing, dispensing, and administering medication. Also, we included all the articles on technical and electronic simulation, surgery, intubation or students or nursing, or technology (serious games, virtual reality, 3D environments).PICO.

Information sources: The search terms "Medical Simulation" [Mesh], "Medication Errors" [Mesh], "Patient safety" [Mesh] were implemented, to be as specific and selective as possible. We searched for all the publications in the Medline database, Web of Science, and Google Scholar from 2000 (when the idea of simulation in healthcare to prevent ME was employed for the first time by the Institute of Medicine (IOM)) to Feb 2022 with only English language-based literatureElectronic databases.

Main outcome(s): Medical errors, Patient safety, Professional performance.

Quality assessment / Risk of bias analysis: The investigators assessed the quality of the each of the included studies. The risk of bias from RCTs was assessed, through Cochrane Collaboration's Tool in seven domains: Adequate Sequence Generation, Allocation Concealment, Blinding of Participants and Personnel, Blinding of Outcome Assessment, Incomplete Outcome Data, Selective Outcome Reporting, Free of Other Bias. The individual domains and overall risk-of-bias judgement were expressed on one of three levels: low risk of bias, unclear risk of bias, and high risk of bias. Based on these factors, the overall quality of evidence was deemed low, moderate, or high risk of bias (details of quality assessment is provided in supplement Table 1). Newcastle-Ottawa Scale (NOS) was used to assess the quality of the cohort studies. NOS score 1-5 were considered as high risk for bias, 6-7 was moderate and score $>7$ was considered low risk of bias.

Strategy of data synthesis: We extracted data by entering them on a computer spreadsheet. Discrepancies were resolved through consensus discussions among investigators. The following data were tried to extract from each eligible study: first author's name, year of publication, location of study, date of study and sample size.

Subgroup analysis: There are no subgroup analysis.

Sensitivity analysis: The results were analyzed using narrative analysis. A textual approach was used to combine and summarize the findings from different studies and subsequently explain the synthesized findings. It was selected as it systematically evaluates and incorporates the results from across the studies and explores the similarities and dissimilarities between the study findings. Since the included studies demonstrated heterogeneity regarding their evaluation criteria and study results, performing a meta-analysis was not considered appropriate, as it would have yielded potentially insignificant and misleading results.

Language: English.

Country(ies) involved: Saudi Arabia.

Keywords: simulation - Medical Error Patient Safety.

Contributions of each author:

Author 1 - Qasem Almulihi.

Author 2 - Asaad Shujaa. 